\title{
Effect of Tacrolimus in Triple Negative Breast Cancer Animal Model
}

\author{
Safiye Aktas, ${ }^{1, *}$ Ayșe Pınar Ercetin ${ }^{1}$, Efsun Kolatan ${ }^{2}$ \\ ${ }^{1}$ Department of Basic Oncology, Dokuz Eylül University Institute of Oncology, Izmir, Turkey. \\ ${ }^{2}$ Department of Laboratory of Animal Science, Dokuz Eylul University School of Medicine, Izmir, Turkey.
}

How to cite this paper: Safiye Aktas, Ayşe Pınar Ercetin, Efsun Kolatan. (2021) Effect of Tacrolimus in Triple Negative Breast Cancer Animal Model. International Journal of Clinical and Experimental Medicine Research, 5(3), 269-277.

DOI: 10.26855/ijcemr.2021.07.007

Received: April 13, 2021

Accepted: May 8, 2021

Published: May 28, 2021

*Corresponding author: Safiye Aktas, Department of Basic Oncology, Dokuz Eylül University Institute of Oncology, Izmir, Turkey.

Email: safiyeaktas@gmail.com

\begin{abstract}
Aim: Triple negative breast cancer has poor prognosis requiring new combination treatments strategies. Structurally resembling the best known mTOR inhibitor of rapamycin and a calcineurin inhibitor, tacrolimus modulates mTOR in the absence of rapamycin with antiproliferative efficacy shown for cancer types like glioblastoma multiforme, hepatocellular carcinoma, and lymphoma, the aim of this study is to assess the efficacy of tacrolimus on tumor cells from an in vitro 4T1 cell line representing triple-negative breast cancer and in a cancer model in experimental animals. Method: 4T1 triple-negative breast cancer cell line was incubated with tacrolimus $(1,10,20$, $30,40,50,100 \mu \mathrm{m})$ for 24,48 and 72 hours. Cell viability percentages were assessed with WST-1 analysis. 14 athymic nude mice with mean weight $25 \mathrm{~g}$ aged 5-7 weeks were injected with 4T1 cells subcutaneously. After $150 \mathrm{~mm}^{3}$ tumor development, animals were randomized into a control group administered physiologic serum and an experimental group administered $10 \mathrm{mg} / \mathrm{kg}$ tacrolimus, on the $1^{\text {st }}$ and $8^{\text {th }}$ days via the intraperitoneal route. On the $14^{\text {th }}$ day, animals were sacrificed and changes in tumor tissue were examined histopathologically and for apoptosis with flow cytometry with annexin- $\mathrm{V}$ and propidium iodide in fresh tissue suspension. Results: The LD50 dose of tacrolimus was identified as $30 \mu \mathrm{M}$. In the triple-negative breast cancer model in experimental animals, the targeted tumor size was reached in 8-12 days. Side effects were not observed from the two doses of tacrolimus administered at a one-week interval. After sacrifice on the $14^{\text {th }}$ day, histopathologic assessment of tumor tissues observed prominent necrosis, necrobiosis and apoptosis in the tumor group compared to the control group. Annexin-V+PI flow cytometry investigation found the early apoptotic effect of tacrolimus was higher compared to late apoptosis and necrosis. Conclusion: Tacrolimus was found to be effective both in vitro and in vivo on triple-negative breast cancer triggering necrosis in addition to apoptosis. Tacrolimus is promising as a treatment choice for the clinical problem of triple-negative breast cancer.
\end{abstract}

\section{Keywords}

Triple-negative breast cancer, experimental animal cancer, mTOR, tacrolimus

\section{Introduction}

Breast cancer is the most common cancer type in women and is second place among all cancer deaths. After lung cancer, it is the cancer type with highest incidence in the world. It can be observed in males, with female cases 100 
times greater than male cases [1-3]. Currently, the majority of breast cancer deaths are not due to primary tumor but related to metastasis and with adjuvant therapies applied to regress metastasis, recurrence can only be reduced by 20\%-30\%. Treatment varies according to tumor size, stage, growth rate and molecular characteristics. The genes p53, c-myc, BRCA1, BRCA2, HER2, and bcl are mainly responsible for carcinogenesis in breast cancer, and are topics of frequent research due to complicated molecular characteristics [4-6].

Among breast cancers with different molecular and biological characteristics, triple-negative (estrogen receptor (ER) negative, progesterone receptor (PR) negative and HER2 negative) breast cancer does not respond to treatment agents targeting these three receptors due to molecular characteristics. As a result, it is a breast cancer type with aggressive characteristics and poor prognosis requiring new combination treatments and agents. Though optimal treatment has survival close to other hormone-positive breast cancer types, the relapse risk is 3-5 times greater than other breast cancer types [7, 8]. 4T1 cells are a mouse-sourced commercial cell line which is defined for triple-negative (ER(-), PR(-) and HER2 nonamplified) and confirmed with immunohistochemistry and chromogenic in situ hybridization in our laboratory. Triple-negative experimental animals are commonly used as breast cancer models.

Signal conduction in cancer cells frequently includes cytoplasmic kinases (like serine/tyrosine kinase) triggering receptor tyrosine kinases (RTK). mTOR is an intracellular serine/threonine protein kinase located at a central point of the intracellular signal cascades. (PI3K)/AKT kinase chain, protein kinase C family (PKC) and mitogen-activated protein kinase (MAPK)/Ras signalization chains are included among the three large signalization paths identified to be important for cancer $[9,10]$. The protein function process of the important signal pathways of $\mathrm{PI} 3 \mathrm{~K} / \mathrm{AKT} / \mathrm{mTOR}$ is closely associated with receptor tyrosine kinases. A variety of RTKs like venous endothelial growth factor (VEGF) receptor (VEGFR), platelet-derived growth factor (PDGF) receptor-a, epidermal growth factor (EGFR), and c-Met may be released from cancer cells. These RTKs use PI3K/AKT/mTOR signal pathways when shaping cancer cell functions. mTOR inhibitors prevent transition from G1 phase to S phase for translocation and cell cycle of required proteins in the cell cycle. As a result, agents inhibiting the PI3K/AKT/mTOR signal pathway have been developed [10-12]. The best known mTOR inhibitors-rapamycin and derivatives-have provided long-term objective tumor response in clinical studies. The rapamycin derivative tacrolimus shows antiproliferative effect inhibiting the mTOR pathway with significant role in cell growth and proliferation. Additionally, it suppresses IL-2 release preventing activation of T and B cells and has immunosuppressive effect. As a result, it is used in transplantation. Due to antiproliferative properties, it is used for treatment of cancer types like multiforme and lymphoma. With no benefit from treatment agents targeting hormone receptors for the very different molecular and biological breast cancer subtype of triple-negative breast cancer, the relapse risk is higher compared to hormone receptor positive breast cancer and it is a cancer type with poor prognosis. As a result, it is a cancer type requiring new combination and targeted treatment agents. There are many studies about the mTOR pathway in breast cancer; however, there is no study in the literature about the efficacy of tacrolimus on tumor load in experimental animals with poor prognosis triple-negative breast cancer [13-15]. Cases developing posttransplant hormone positive breast cancer have been administered tacrolimus with the aim of immunosuppressive effect along with anticancer endocrine treatment. TAMRAD and BOLERO-2 studies found administration of endocrine treatment with tacrolimus increased progression-free survival [16]. In metastatic breast cancer resistant to aromatase inhibitors, addition of mTOR inhibitor increased survival and quality of life. Additionally, the mTOR pathway was shown to be activated in breast cancer resistant to hormonal treatment and chemotherapy [17]. The BOLERO-3 clinical study found addition of vinorelbine and mTOR inhibitor everolimus to anti-HER2 treatment for transtuzumab-resistant breast cancer increased survival [17]. Publications from early phase studies of mTOR inhibitors with various chemotherapeutics for metastatic triple-negative breast cancer have begun to be included one-by-one in the literature [18]. The use of mTOR inhibitors in breast cancer has still not entered routine procedures. Triple-negative breast cancer displays a heterogeneous clinic. Very poor prognosis may be shown for cases resistant to chemotherapy, especially. mTOR inhibitors are a new treatment candidate in this field.

It is necessary to interrogate new agents which may be effective for the triple-negative subgroup of breast cancer, without a candidate for hormone treatment or targeted treatment. In our research, we aimed to assess the effect of tacrolimus alone on a triple-negative breast cancer experimental animal model. mTOR inhibitors have begun to be used commonly for treatment of cancers, apart from their use after organ transplantation. As a result of this study, it is expected the efficacy of tacrolimus on an in vivo model of triple-negative breast cancer will be identified and minimum toxic effect will be observed in other organs. Based on this knowledge, the aim of this study is to research the efficacy of the new-generation mTOR inhibitor tacrolimus on tumor load in an experimental model of triple-negative breast cancer, with high relapse risk and poor prognosis. 


\section{Material and Method}

The study received permission from Dokuz Eylül University Multidisciplinary Laboratory Animal Experiments Local Ethics Committee.

\subsection{Cell Culture}

4T1 triple-negative breast cancer cells (ATCC CRL-2539) were incubated in RPMI 1640 medium (1\% L-glutamine and $1 \%$ penicillin/streptomycin) containing $10 \%$ fetal bovine serum at $37^{\circ} \mathrm{C} 5 \% \mathrm{CO} 2$. Cells were removed from the flask surface with trypsin-EDTA solution when approximately $90 \%$ confluence was reached and placed in a 96-well plate with 6 wells/group and 5,000 cells per well and left for 24 hours. Tacrolimus was applied with different doses (from 1-100 $\mu \mathrm{M}$ ). The plate was placed in $37^{\circ} \mathrm{C} 5 \% \mathrm{CO} 2$ incubator for 24 hours. When the incubation period ended, cell viability was recorded with WST-1. LD50 dose was optimized and administered in animal experiments.

\subsection{Assessment of cell proliferation with WST-1}

The 96-well plate containing cells had an empty well containing medium left as blind. For each well in the 100 $\mu \mathrm{l} /$ well cell, $10 \mu \mathrm{l} /$ well WST-1 cell proliferation reactive was added (1:10 dilution). Cells were left for 2 hours for incubation in a $37^{\circ} \mathrm{C} 5 \% \mathrm{CO} 2$ incubator. After the plate was shaken for $1 \mathrm{~min}$ with a shaker, it was read at 420-480 $\mathrm{nm}$ with an ELISA reader. Reference wavelength was chosen as more than $600 \mathrm{~nm}$ (630 nm). Mean absorbance in the control group was accepted as $100 \%$ viability, and other absorbances were compared to this to record cell viability percentage.

\subsection{Mouse Xenograft Model}

Five-seven weeks old female nude mice were purchased from Kobay Experimental Animal Laboratory for the study. Animals were housed in Dokuz Eylül University Multidisiplin Laboratory Experimental Animal Facility where housing conditions were standard $\left(20 \pm 2^{\circ} \mathrm{C}, 55 \%\right.$ relative humidity) with 12 hour light/dark cycles (lights on from 07:00 to 19:00). Animals kept in IVC cages. Sterilized food and water were available ad libitum. The study begun one week after the animals brought to the facility.

Tumor development was maintained by injecting 4T1 triple negative breast cancer cell line of $1 \mathrm{x} 106 \mathrm{cell} / \mathrm{mL}$ via the SC route to nude mice. When developed tumor volume reached $150 \mathrm{~mm} 3$, mice randomized into two groups $(\mathrm{n}=7)$.

\section{Experimental Animal Groups}

Two groups were formed by random animal selection. In each group there were 7 animals.

Group I ( $\mathrm{n}=7)$ : physiologic solution applied control group

Group II (n=7): tacrolimus applied group

Chemicals

Tacrolimus: prograf ampule $(5 \mathrm{mg} / \mathrm{ml})$

Two dose of tacrolimus was injected during the study. Each dose of $10 \mathrm{mg} / \mathrm{kg}$ tacrolimus was given by weight via the IP route.

\section{Experimental animal applications}

Tacrolimus and physiologic solution applications

Mice in group I received $0.5 \mathrm{ml}$ physiologic solution via the IP route on 1st and 8th days (the days group II mice receive tacrolimus).

Group II animals received $10 \mathrm{mg} / \mathrm{kg}$ tacrolimus and necessary amount of physiologic solution for making the injected volume of the solution even for all the animals $(0.5 \mathrm{ml})$ in the study. The solution was given via the IP route on 1st and 8th days.

\section{Human endpoints}

In case of sepsis development, decreased responses to stimuli, more than $15 \%$ of weight loss or suggested by the veterinary, mice were euthanized by ether anesthesia. Animals were observed once a day for their behavior in home cage and weighted once in two days. To keep the planned animal number in the study, new animals were added following the procedures to set the model as mentioned. 


\section{Cautions taken for the researchers}

Tacrolimus solution was prepared daily under sterile conditions just before the application, by researchers wearing surgical gloves, protective goggles and sterile laboratory coat. After the application, the leftover drug was destroyed in DEU chemotherapy unit.

\section{Storage of Serum Samples}

At the end of the study (on 14th day) blood samples were taken via cardiac puncture of all mice with insulin injector when animals were under general anesthesia. Collected blood was transferred to plain blood tube. Serum was collected after centrifuged for $15 \mathrm{~min}$ at $3,000 \mathrm{rpm}$ and stored at $-80^{\circ} \mathrm{C}$ for future studies. After blood withdrawal, tissues and organs were dissected.

\section{Sacrification of mice, tissue and organ dissections}

Mice were sacrificed 14 days after the first tacrolimus (group I) and equal volume of physiologic solution injections (group II). All mice were sacrificed under ether anesthesia. Mice tumor tissues and organs were dissected. The tumor was resected, all three cavities were opened and explored. Half of the tumor and organs were immediately stored at $-80^{\circ} \mathrm{C}$ for future biomedical analysis. Half of brain, kidney, lung, heart, liver and tumor tissue was fixated in formalin and submerged in paraffin after tissue monitoring procedures.

Sections with 5 micrometer thickness prepared from paraffin blocks were placed on polylysine slides, stained with hematoxylin eosin for pathologic examination and apoptosis staining with the TUNEL method. Assessment performed counts of 1,000 cells in 5 different areas and recorded apoptosis as mean percentage. Single cell suspensions were obtained from the remaining fresh tumor tissue. Flow cytometric apoptosis count was performed with annexin-V. In this way, apoptosis analysis was completed for tissue and cells with two methods.

\subsection{Assessment of apoptosis in tumor tissue in mice with annexin $\mathrm{V}$ and flow cytometry}

Some of the dissected tumor tissues were passed through a filter with pores with 50 micrometer diameter after homogenization in a laminar-flow cabinet and single cell suspensions were obtained. The suspended cells obtained from tumor tissue was placed in a $15 \mathrm{~mL}$ falcon tube and centrifuged at 1,200 rpm for $5 \mathrm{~min}$. After removing the supernatant, the $1 \mathrm{x}$ concentration, prepared by dissolution using the $10 \mathrm{x}$ binding buffer from the pellet kit, was resuspended in $100 \mu \mathrm{l}$ buffer and transferred to a polystyrene $5 \mathrm{~mL}$ flow cytometric test tube. To obtain the correct gating setting for this analysis, the same sample was placed in a tube without staining, another tube had only $5 \mu \mathrm{l}$

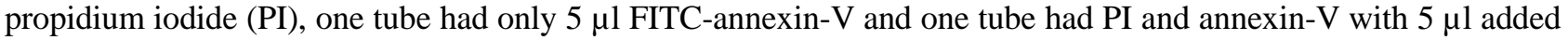
to each and incubated in the dark at room temperature for 15 minutes. At the end of incubation, the tube had $400 \mu \mathrm{l}$ binding buffer added and flow cytometry analysis was performed. After appropriate gating was performed, only annexin- $\mathrm{V}$ stains were used to define early apoptotic, while both annexin- $\mathrm{V}$ and PI were used to define late apoptotic or necrotic cells. These percentage values had comparative calculations performed according to the initial cell counts.

\subsection{Apoptosis analysis with TUNEL in paraffin sections}

Sections were left in an incubator at $60^{\circ} \mathrm{C}$ overnight. After sections cooled, they were left for 2 x 30 min in xylene. Necessary information was written on the slide in pencil. Sections were left in $96 \%, 80 \%, 70 \%$ and $60 \%$ ethyl alcohol for 2 minutes each and then washed in PBS for 5 minutes. The TdT labeling mixture was prepared by mixing TdT buffer, biotin d-UTP and TdT in the ratio of 90:5:5. Then cells were incubated with $50 \mu$ of TdT labeling mixture each for $60 \mathrm{~min}$ at room temperature. TB (10x) buffer was diluted with distilled water to 1:10 dilution and then slides were dipped in the obtained TB (1x) buffer and left for $5 \mathrm{~min}$ at room temperature and the TdT reaction ended. Sections were washed 4 times in PBS for 2 minutes each time. Blocking solution and PBS were mixed in the ratio 1:3 to prepare blocking buffer. Sections were incubated at room temperature with $50 \mu$ blocking solution each for $20 \mathrm{~min}$ and then removed from the buffer. Then avidin-FITC and the blocking buffer prepared in the previous step were mixed in 1:9 ratio and sufficient avidin-FITC solution for $50 \mu \mathrm{l}$ per sample was prepared. Samples were incubated in $50 \mu \mathrm{l}$ avidin-FITC solution in the dark at room temperature for $30 \mathrm{~min}$. Then they were washed 3 times with PBS for 5 min each in the dark. Slides were covered with glycerin and investigated with a fluorescent microscope.

\subsection{Statistical analysis of data}

Data are given as mean \pm standard deviation. Statistical analysis of the research results used the SPSS for Windows statistical program version 15.0 at $\mathrm{p}<0.05$ significance level. Comparisons between groups used the Kruskal 
Wallis and then the Mann-Whitney U test.

\section{Results}

\subsection{Cell proliferation results assessed with WST-1}

Cell proliferation was assessed with WST-1 after 24, 48 and 72 hours of incubation with 1, 10, 20, 30, 40, 50 and $100 \mu \mathrm{M}$ doses of tacrolimus (Figure 1). These in vitro assessment results showed that tacrolimus reduced cell viability in dose-linked fashion and this cytotoxic effect did not vary linked to duration. The LD50 dose of tacrolimus was identified as $30 \mu \mathrm{M}$ (Figure 1).

\subsection{Sacrifice results of experimental animals}

When tumor development reached $10 \mathrm{~mm}$ (Figure 2), tumor diameter was measured each day after tacrolimus or PS administration. There was no statistical difference in the mean tumor diameters. The reason for this is interpreted as the end point of the experiment having short follow-up duration. Though tumor tissue displayed necrosis and apoptosis with tacrolimus, the resorption of this cellular waste had not happened yet.

Tumor tissue generally had solid tumor pattern. Vascularization was noted to be more pronounced on the periphery. The tumor was invasive of surrounding muscle and connective tissue, but was observed to have good margins. Mitotic activity was high. The group administered tacrolimus had clear apoptosis and necrosis/necrobiosis noted (Figure 3).

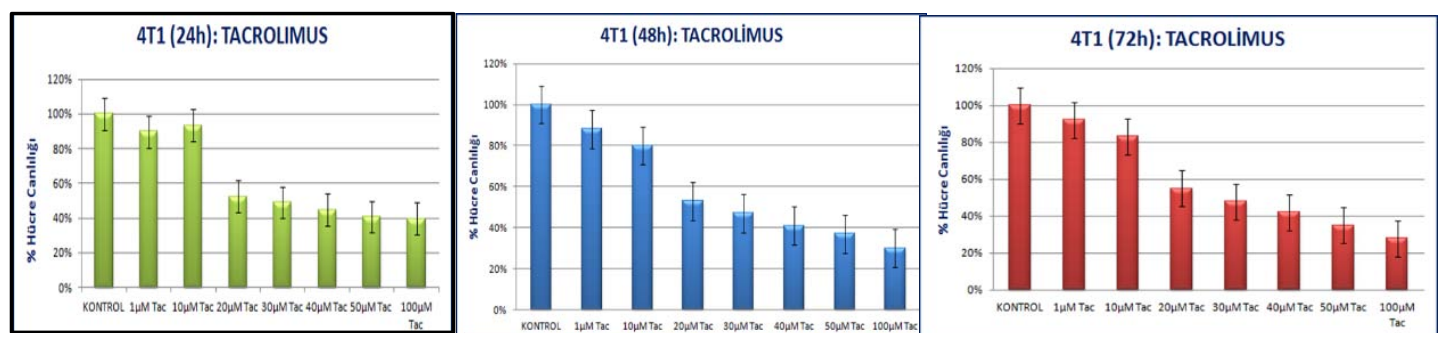

Figure 1. Cell proliferation after 24, 48 and 72 hours of incubation with 1, 10, 20, 30, 40, 50 and $100 \mu \mathrm{M}$ doses of tacrolimus. Dose dependent cell viability reduction, not time. The LD50 dose of tacrolimus was identified as $30 \mu \mathrm{M}$.

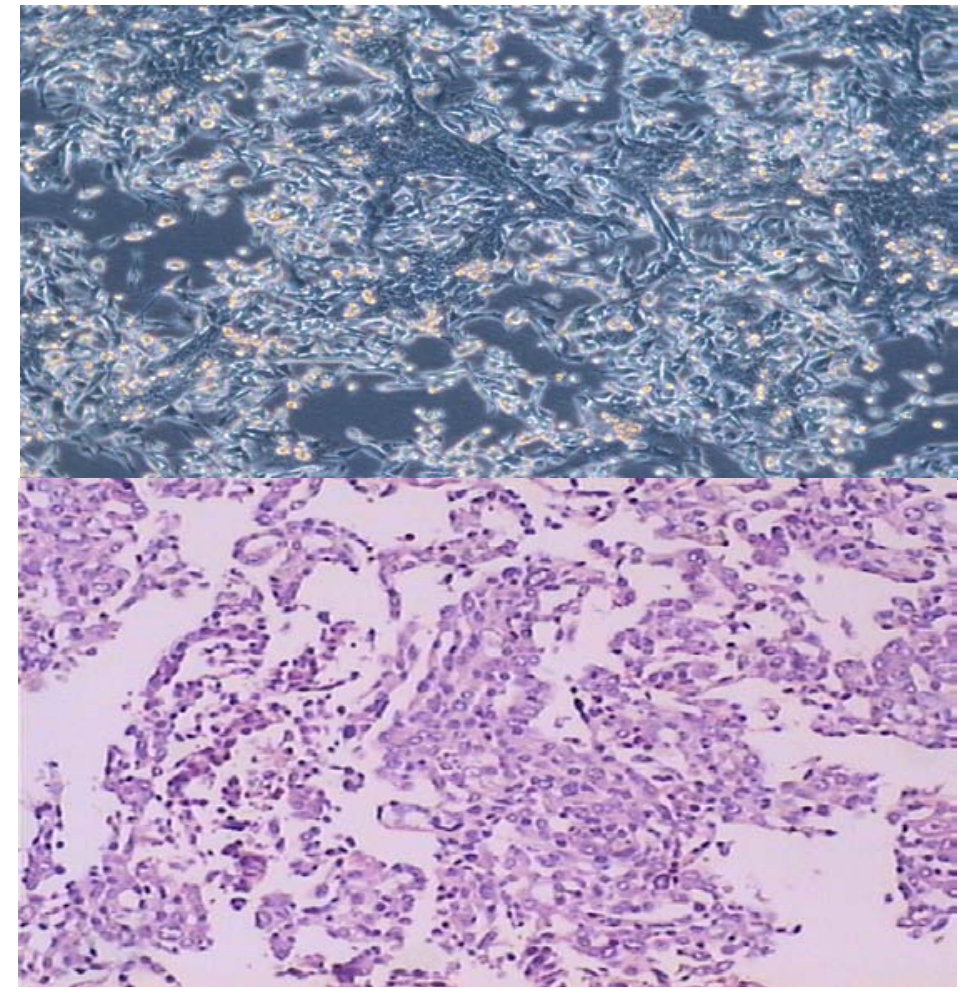

Figure 2. 4T1 cells in flask (left) and H\&E stained cell block appearence (right) (X100). 


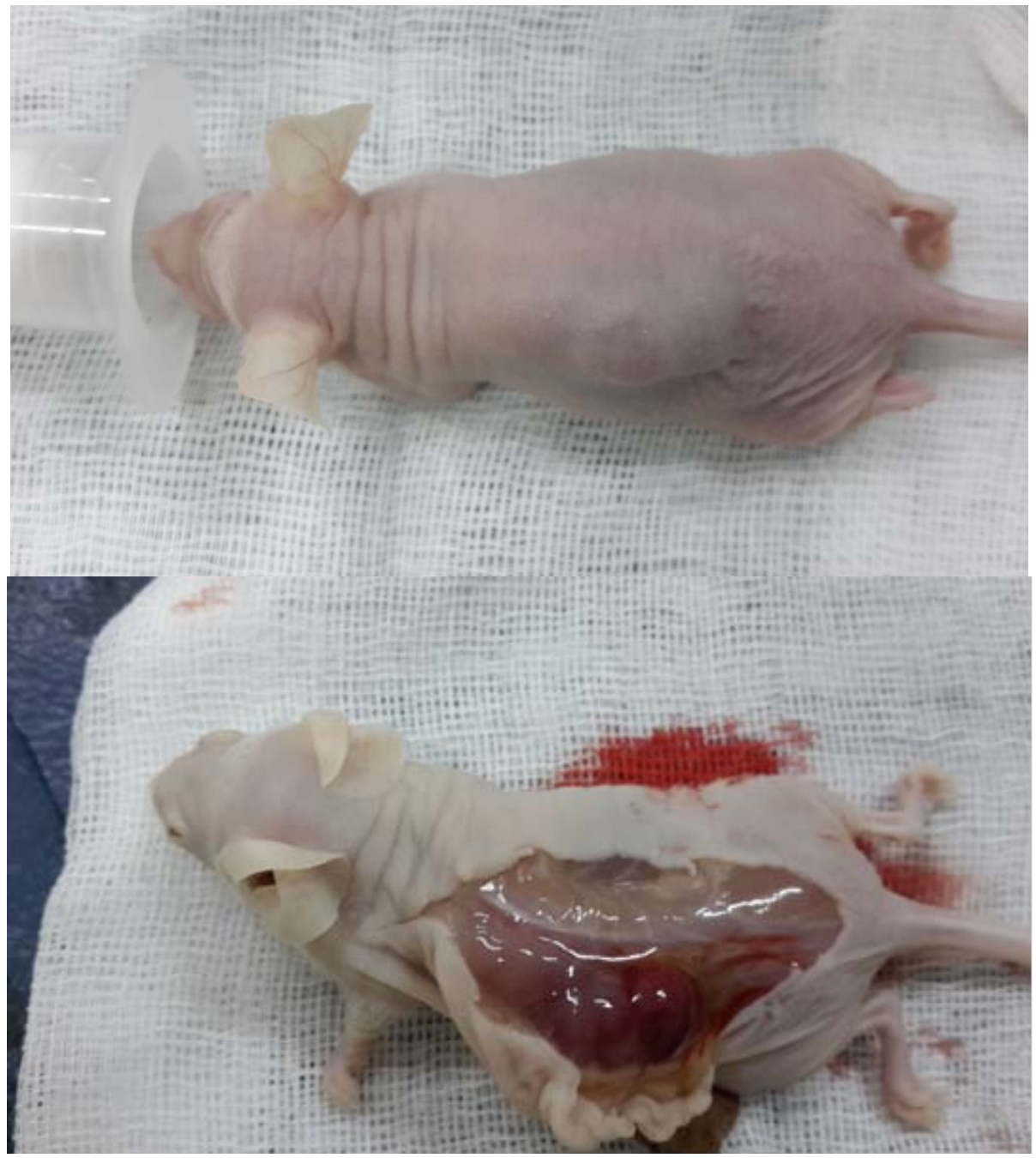

Figure 3. Nude mice with a subcutanous noduler solid tumor on the left back side in injection locus.

\subsection{Apoptosis results for tumor tissue in mice with annexin $\mathrm{V}+$ propidium iodide and flow cytometry}

At the end of the study, fresh tumor samples collected during sacrifice of animals were used to obtain single cell suspensions and flow cytometric apoptosis assessments were made with gating taken from the control group. Thus, early apoptosis, late apoptosis, necrosis and live cell percentages were obtained (Table 1).

\subsection{Necrosis/necrobiosis results on paraffin sections and Apoptosis analysis results with TUNEL}

Area percentages of necrosis/necrobiosis were calculated with hematoxylin/eosin staining on paraffin sections (Figure 4). TUNEL staining counted positive cells per 5000 cells and percentage values were recorded (Table 2).

Table 1. Apoptosis stages in tumor cell suspentions of control and tacrolimus groups determined by flow cytometer AnnexinV+PI. Tacrolimus did not show statistically significant difference $(p>0.05)$

\begin{tabular}{ccccccccccc}
\hline$\%$ & Mouse no & 1 & 2 & 3 & 4 & 5 & 6 & 7 & mean \\
\hline \multirow{2}{*}{ Control } & Early apoptosis & 49.2 & 30 & 19.6 & 36.3 & 67.6 & 47.6 & 51.3 & 40.5 \\
& Late Apoptosis & 12.8 & 3.2 & 3.2 & 9.8 & 12.7 & 21.2 & 22.6 & 8.3 \\
& Necrosis & 5.3 & 1 & 0.2 & 8.2 & 1.1 & 2.0 & 8.4 & 3.2 \\
\multirow{3}{*}{ Tacrolimus } & Early apoptosis & 12.8 & 73 & 57.4 & 24 & 16.1 & 22.8 & 43.1 & 43.8 \\
& Late Apoptosis & 12.8 & 5.5 & 7.3 & 4.9 & 4.6 & 10.1 & 8.1 & 7.61 \\
& Necrosis & 4.6 & 0 & 14.1 & 0.8 & 1.9 & 0.2 & 3.8 & 3.63 \\
\hline
\end{tabular}


Table 2. Necrosis and Apoptosis ratios in tumor tissues of control and tacrolimus groups determined by histopathology and TUNEL assay. Tacrolimus showed statistically significant increased apoptosis and necrosis $(p=0.01$, for both apoptosis and necrosis)

\begin{tabular}{ccccccccccc}
\hline$\%$ & Mouse no & 1 & 2 & 3 & 4 & 5 & 6 & 7 & mean \\
\hline \multirow{2}{*}{ Control } & apoptosis & 9.6 & 4.2 & 4.1 & 8.2 & 10.4 & 11.0 & 7.8 & 7.9 \\
& necrosis & 10 & 5 & 5 & 6 & 10 & 10 & 6 & 7.43 \\
\multirow{3}{*}{ Tacrolimus } & apoptosis & 65.8 & 26.7 & 80.2 & 64.3 & 31.0 & 28.6 & 37.1 & 47.67 \\
& necrosis & 52 & 35 & 70 & 65 & 40 & 45 & 35 & 48.86 \\
\hline
\end{tabular}

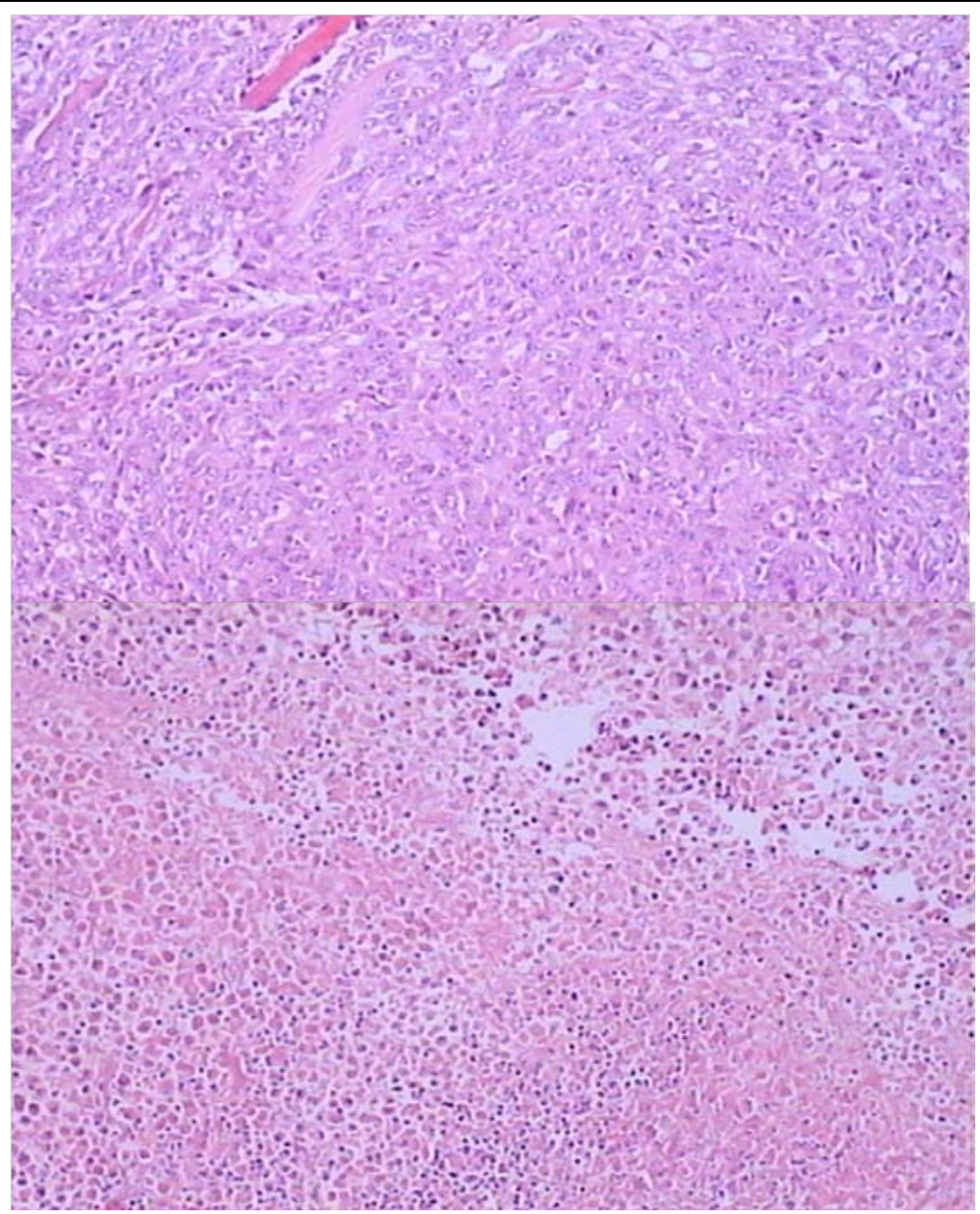

Figure 4. Histopatologic microscopic photos of tumor sections (H\&Ex 100) Control group on the left viable solid tumor highly vascularized. On the right Tacrolimus group, tumor area with prominent necrosis and apoptosis.

\subsection{Statistical Analysis Results}

Nonparametric tests were used to compare the control group with the tacrolimus group in SPSS 15.0. The tumors in the tacrolimus group appeared to have more apoptosis (TUNEL) and necrosis/necrobiosis compared to the control group ( $\mathrm{p}=0.001$ ) (Table 2). The mean apoptosis in the control group was $7.9 \%$, while mean necrosis/necrobiosis was $7.43 \%$. In the tacrolimus group, mean apoptosis was $44.65 \%$, while mean necrosis/necrobiosis was $48.33 \%$. When the control group and tacrolimus group are statistically compared with the nonparametric Mann-Whitney $\mathrm{U}$ test, the tacrolimus group was found to have higher apoptosis count $(\mathrm{p}=0.001)$ and necrosis count $(\mathrm{p}=0.001)$.

\section{Discussion}

Breast cancer is the most commonly observed cancer type in women and is second place among all cancer deaths. Triple-negative [estrogen receptor (ER) negative, progesterone receptor (PR) negative and HER2 negative] breast 
cancer does not respond to treatment agents targeting these three receptors due to molecular features. As a result, the efficacy of many new agents has been researched. This study with the same aim assessed the effect of tacrolimus, a calcineurin inhibitor associated with the mTOR pathway with immunosuppressive features effectively used mostly after organ and stem cell transplants, on triple-negative breast cancer. In vitro experiments showed the inhibitory effects of tacrolimus on cell proliferation, and later this efficacy was assessed in a nude mice experimental animal model. Experimental groups comprised seven control animals and seven animals administered tacrolimus. Nearly two weeks after subcutaneous cell administration, the first target in the study of tumor formation was positively achieved. After tumor formation, the control group were not administered tacrolimus, while the other seven animals were administered tacrolimus; however, one animal died before the study was completed and was excluded. Before assessing the data obtained as a result of this study, there were not many studies encountered assessing the efficacy of tacrolimus on cancer cells in the current literature. This study is important in terms of unique value as no previous study has been completed about triple-negative breast cancer.

When the literature is assessed, there appear to be current studies about the antitumor efficacy of tacrolimus alone and/or in combination on pancreas, bladder, prostate, hepatocellular carcinoma and ovarian cancer. Constantinescu et al. assessed the effect of tacrolimus and sirolimus on mitochondria-mediated apoptosis in pancreatic cells. The study showed these two agents induced the mitochondrial apoptotic route in normal pancreas cells causing pancreatic cell dysfunction with a variety of viability tests and gene expressions [7]. Wang and team assessed the paclitaxel and tacrolimus combination on ovarian cancer based on the consideration that combinations of chemotherapeutic agents are important to overcome multiple resistance. However, they administered this combination in polyethylene glycol-polyepsilon caprolactone micelles due to low solubility of anticancer drugs in water. In conclusion, this combination showed clear increase in G2/M arrest and apoptosis in paclitaxel-sensitive ovarian cancer cell lines [19].

The effect of exposure to immunosuppressive calcineurin inhibitors in solid organ cancers occurring after liver transplant is not fully known. Considering this, Carenco et al. assessed tacrolimus in terms of this risk and showed there was $\geq 3$ years exposure to tacrolimus in 216 patients developing solid tumors after transplant and that the levels in blood were high in these patients. In conclusion, they emphasized the need to use tacrolimus carefully after liver transplant [5]. The functional effect of active t cell nuclear factor (NFATc1) with an important role in regulating the immune response in development of bladder cancer is not known. Kawahara et al. assessed the effect of tacrolimus and cyclosporin on bladder cancer and showed NFATc- 1 transcription factor caused a reducing trend in regulation and as a result, cell viability/colony formation, cell migration/invasion and MMP-2 and MMP-9 expression reduced [11]. Similarly, the same team assessed this effect in prostate cancer. As a result of the study, NFATc-1 played an important role in development of prostate cancer, as a result cyclosporin A and tacrolimus and NFATc-1 inactivation may be a potential therapeutic approach to hormone-sensitive and castration-resistant prostate cancer [12]. Kawahara et al. in another assessment with the same drugs observed that cyclosporin A and tacrolimus reduced NFATc-1 expression and transcriptional activity in immortalized normal epithelial cells. Additionally, p53 and p21 expression increased, which is important for tumorigenesis in bladder cancer, and expression of cyclin D1, cyclin D3 and cyclin E oncogenes reduced. In this way, they revealed that cyclosporin A and tacrolimus inhibited bladder cancer tumorigenesis in urothelial cells [13]. Zhu et al. showed that tacrolimus increased in vivo CXCR4/SDF-1 $\alpha$ expression and progressed hepatocellular carcinoma [23]. However, according to the study by Capone et al. doxorubicin and tacrolimus induced apoptosis with the synergistic effect in hepatocellular carcinoma cell lines, reduced autophagic activation, reduced proinflammatory cytokines and showed antitumor properties [6].

As in these studies, in our study the antitumor effect of tacrolimus on triple-negative breast cancer was assessed and cell viability, apoptosis and necrosis amounts in tumor tissue induced in experimental animals was revealed with a variety of methods. In accordance with most studies, tacrolimus reduced the breast cancer cell proliferation in triple-negative breast cancer and this occurred by increased apoptosis but not necrosis.

\section{Conclusion}

Tacrolimus was found to be effective on triple-negative breast cancer in vitro and in vivo. This effect was shown by triggering necrosis in addition to apoptosis especially. Tacrolimus is promising as a treatment choice for the clinically significant problem of triple-negative breast cancer.

\section{Acknowledgments}

This project was supported by the Scientific and Technological Research Council of Turkey (TÜBİTAK) 1002 projects with project number 215 S048. 


\section{References}

[1] Al-Hajj, M., Wicha, M. S., Benito-Hernandezt, A., Morrison, S. J., Clarke, M. F. (2003). Prospective identification of tumorigenic breast cancer cells. PNAS, 100(7): 3983-88.

[2] Hassan, B. A., Yusoff, Z. (2011). Negative Impact of Chemotherapy on Breast Cancer Patients QOL-Utility of Antiemetic Treatment Guidelines and the Role of Race. Asian Pacific J Cancer Prev, 1523-1527.

[3] Russell, C. A. (2014). Personalized medicine for breast cancer: it is a new day! Am J Surg, 207: 321-5.

[4] Gaudet, M. M., Press, M. F., Haile, R. W., Lynch, C. F., Glaser, S. L., Schildkraut, J., Gammon, M. D., Douglas Thompson, W., Bernstein, J. L. (2011). Risk factors by molecular subtypes of breast cancer across a population-based study of women 56 years or younger. Breast Cancer Res Treat., 130(2): 587-597.

[5] Marshall, J. C., Collins, J. W., Nakayama, J., Horak, C. E., Liewehir, H. J., Steinberg, S. M., Albaugh, M., Vidal-Vanaclocha, F., Palmieri, D., Barbier, M., Murone, M., Steeg, P. S. (2012). Effect of inhibition of the lysophosphatidic acid receptor 1 on metastasis and metastatic dormancy in breast cancer. J Natl Cancer Inst., 104(17): 1306-19.

[6] Bernstein, L., Lacey, J. V. Jr. (2011). Receptors, associations and risk factor differences by breast cancer subtypes: positive or negative? J Natl Cancer Inst., 103: 451-453.

[7] Boyle, P. (2012). Triple-negative breast cancer: epidemiological considerations and recommendations. Ann Oncol, 23 Suppl 6: vi7-vi12.

[8] Hudis, C. A. (2011). Gianni L. Triple-negative breast cancer: an unmet medical need. Oncologist, 16: 1-11.

[9] Yang, H., Rudge, D. G., Koos, J. D., Vaidialingam, B., Yang, H. J., Pavletich, N. P. (2013). mTOR kinase structure, mechanism and regulation. Nature, 497: 217-23.

[10] Shaw, R. J., Cantley, L. C. (2006). Ras, PI(3)K, and mTOR signalling controls tumor cell growth. Nature, 441: 424-30.

[11] Zaytseva, Y. Y., Valentino, J. D., Gulhati, P., Evers, B. M. (2012). mTOR inhibitors in cancer therapy. Cancer Letters, 319: $1-7$.

[12] Yu, K., Toral-Barza, L. (2012). Biochemical and pharmacological inhibition of mTOR by rapamycin and an ATP-competitive mTOR inhibitor. Methods Mol Biol, 821: 15-28.

[13] Maguire, O., Tornatore, K. M., O'Loughlin, K. L., Venuto, R. C., Minderman, H. (2013). Nuclear translocation of nuclear factor of activated T cells (NFAT) as a quantitative pharmacodynamic parameter for tacrolimus. Cytometry A, 83: 1096-104.

[14] Augusto, J. F., Subra, J. F., Onno, C., Villemain, F., Croue, A., Dussaussoy, C., Picquet, J., Coeffic, B., Sayegh, J. (2013). Long-term maintenance immunosuppressive regimen with tacrolimus monotherapy. Ann Transplant, 18: 368-77.

[15] Siamakpour-Reihani, S., Caster, J., Bandhu, Nepal, D., Courtwright, A., Hilliard, E., Usary, J., Ketelsen, D., Darr, D., Shen, X. J., Patterson, C., Klauber-Demore, N. (2011). The role of calcineurin/NFAT in SFRP2 induced angiogenesis--a rationale for breast cancer treatment with the calcineurin inhibitor tacrolimus. PLoS One, 2011, 6(6): e20412.

[16] Jeong, I. J., Lee, S. G., Kim, Y. H., Ko, B. S., Lee, J. W., Son, B. H., Ahn, S. H., Kim, H. J. (2017). Characteristics and prognosis of breast cancer after liver or kidney transplantation. Breast Cancer Res Treat, https://doi.org/10.1007/s10549-017-4504-1.

[17] Sendur, M. A., Zengin, N., Aksoy, S., Altundag, K. (2014). Everolimus: a new hope for patients with breast cancer. Curr Med Res Opin., 30(1): 75-87.

[18] Basho, R. K., Gilcrease, M., Murthy, R. K., Helgason, T., Karp, D. D., Meric-Bernstam, F., Hess, K. R., Herbrich, S. M., Valero, V., Albarracin, C., Litton, J. K., Chavez-MacGregor, M., Ibrahim, N. K., Murray, J. L. 3rd, Koenig, K. B., Hong, D., Subbiah, V., Kurzrock, R., Janku, F., Moulder, S. L. (2017). Targeting the PI3K/AKT/mTOR Pathway for the Treatment of Mesenchymal Triple-Negative Breast Cancer Evidence From a Phase 1 Trial of mTOR Inhibition in Combination With Liposomal Doxorubicin and Bevacizumab. JAMA Oncol., 3: 509-515. 